\title{
Caring for Self while Caring for Others: Impact of Wearable Health Monitoring Devices on Self-care of Family/Friend Caregivers of Individuals Living with a Dementia
}

\author{
R. McCloskey ${ }^{1}$, L. Skerry ${ }^{2}$, L. Keeping-Burke ${ }^{3}$, A. Donovan ${ }^{4}$, C. Donovan ${ }^{5}$, and E. \\ Scheme ${ }^{6}$ \\ ${ }^{1,3}$ Department of Nursing \& Health Sciences, University of New Brunswick, Canada \\ ${ }^{2}$ Research Service, Horizon Health Network, Saint John, New Brunswick, Canada \\ ${ }^{4,5}$ Loch Lomond Villa, Saint John, New Brunswick, Canada \\ ${ }^{6}$ Faculty of Engineering, University of New Brunswick, Canada \\ ${ }^{1}$ Rose.McCloskey@unb.ca, ${ }^{2}$ Leanne.Skerry@HorizonNB.ca \\ 3lkeeping@unb.ca, ${ }^{4}$ Alicia.Donovan@unb.ca \\ ${ }^{5}$ Cdonovan@lochlomondvilla.com, ${ }^{6}$ Eric.scheme@unb.ca
}

\begin{abstract}
Dementia is a leading cause of disability and dependency and directly impacts family caregivers. Caregivers often neglect their health and well-being while caring for a loved one; thereby, placing themselves at risk for adverse health outcomes. Identifying ways to promote well-being among family caregivers is necessary so they can continue in their caregiver role while mitigating ill health effects. This study explored the impact of wearable health monitoring devices on caregivers' engagement with their own self-care needs. Findings provide evidence for the use of a digital health platform in promoting health in caregivers of individuals living with dementia.
\end{abstract}

Keywords: Dementia, Caregivers, Technology, Self-care

\section{Introduction}

One of the greatest public health issues facing the world today is Alzheimer's disease and related dementias. Dementia is a leading cause of disability and dependency and has significant physical, psychological, and economic effects on individuals impacted [1]. About 50 million people worldwide have a diagnosis of dementia with an additional 10 million new cases identified each year [2]. More alarming, there are estimates that the number of people living with dementia across the globe will triple by 2050 [3]. Dementia not only affects those individuals living with the disease, but it also has impacts on families. Family caregivers provide an average of 26 hours of unpaid care each week [4], have high information and support needs [5][6], and experience difficulties navigating community resources designed to address their needs [7]. Given these findings, it is not surprising that many individuals living with or caring for somebody with dementia report high levels of unmet needs [5].

While finding a cure and improving the management of people living with dementia is a priority for researchers and clinicians alike, it is equally important is a need to support family

Article history:

Received (July 3, 2019), Review Result (September 5, 2019), Accepted (November 7, 2019) 
caregivers who play a pivotal role in caring for these individuals. This need is vital because the majority of persons with dementia live at home under the care and supervision of family [4].

\section{Literature review}

The literature identifies a range of services and supports that are available in the community to assist those caring for somebody living at home with dementia. These family/friend supports include resources such as support groups, domestic assistance or help with managing the home, nursing care, personal care services, respite care, and educational programs [8]. Despite the wide range of supports available, caregiving resources are often underutilized, even when these services are deemed highly relevant [9][10]. Reasons for not accessing support include a lack of awareness of existing services and/or not knowing how to access them, no perceived need, previous negative experiences, perceptions of poor quality, a sense of responsibility and obligation towards the loved one, a concern for privacy and autonomy, and embarrassment [11][12][13].

Caregivers of persons with dementia who do not access community services report higher levels of depression [14], role resentment [15], and anger towards their care recipient [16] in comparison with caregivers who do access services. Caregivers who use community support also anticipate being able to care for their loved one at home for longer periods than caregivers who do not access services [17]. Nonetheless, community support is often underutilized by family caregivers. One study reports up to $80 \%$ of caregivers of people with dementia do not access community services or only do so during a crisis [14]. A recent scoping review on home-dwelling individuals with dementia and their carers identified five categories of interventions used to improve the use of community-based resources: i. referral enhancing interventions aimed at directing people to use services that are perceived to be beneficial for them; ii. awareness and information interventions that address the population's lack of knowledge about available resources; iii. monetary support interventions that address user fees and private payments for services or support; iv. inpatient-focused interventions that capitalize on hospital admissions to promote the use of services; and, v. case management interventions that involve a key person overseeing and coordinating care delivery for individuals and their families [18].

Health and social policies in most countries emphasize the importance of people with dementia remaining at home for as long as possible, but this ideal is contingent upon capable and willing family caregivers [1]. Yet, research shows that family/friend caregivers often neglect their health and well-being while caring for their loved ones [19]. For instance, family/friend caregivers tend to experience decreased quality of life, sleep problems [20], increased stress levels, and an elevated risk of developing depressive disorders in comparison with non-caregivers [21]. Unfortunately, adverse effects of family/friend caregiving (i.e., the burden) are frequently hidden behind the health of the individual diagnosed with dementia [22]. While unpaid family caregivers provide an essential service to both their loved ones and the health care system, the burden and stress they experience as a direct result of caregiving place them at risk for developing or worsening health problems [19][23]. Health promotion for family caregivers should therefore be a priority, not only because the care of their loved one depends on it, but because caregivers are at risk of becoming patients themselves.

Identifying ways to assist caregivers to continue in their roles while mitigating their ill health effects are necessary to ensure individuals living with dementia can remain at home for as long as possible [24]. Technological-based solutions hold promise as an attractive and 
convenient alternative to face-to-face interventions to deliver education and support for family/friend caregivers [24][25][26]. Results from a systematic review by Boots and colleagues [24] suggest that internet interventions for family/friend caregivers of persons with dementia may have positive effects on caregiver well-being, provided that these interventions are tailored to the individual and consist of multiple components. Specifically, interventions that combined educational materials, strategies for dealing with memory loss, and a means to connect with other caregivers have beneficial effects on caregiver preparedness, burden, stress, depression, and self-efficacy [24][27][28].

Wearable health monitoring devices have demonstrated promise as a means for promoting individual awareness and engagement in achieving personal goals [29]. Mobile technological advances, particularly those with health promotion and monitoring capabilities, create opportunities to empower caregivers to make informed decisions about their daily activities and health practices. The new generation of consumer devices allows for non-invasive monitoring and collection of health-related data such as activity level, caloric intake, blood pressure, and sleeping patterns. How the integration of these devices, along with qualitative health observations, translates to beneficial outcomes for caregivers of persons with dementia remains unknown. Given the dramatic increase in the use of technology among older adults over the last decade [30], investigations of internet-based solutions as a source of support and education for family/friend caregivers are warranted.

This paper reports on a study that explored the experiences of caregivers of persons living with dementia who used wearable health monitoring devices in conjunction with a mobile platform (iWellness; Inflection Point Healthcare, Canada) to support their health-related behaviors. The iWellness platform consists of multiple components that have the potential to engage and support family/friend caregivers by offering informational resources about dementia, social-support components, and tracking of health information.

\section{Methods}

\subsection{Intervention}

The iWellness (Inflection Point Healthcare, Canada) digital health platform was designed for this study to specifically facilitate the management, aggregation, and presentation of participant health data. The platform allowed participants to collect a multitude of personal health information from their choice of supported consumer-grade devices into one repository. Data (i.e., steps and sleep) from the wearable health monitoring devices worn by participants (e.g., Fitbit product, blood pressure monitor) were automatically uploaded to the iWellness application. Additional health data that could be manually entered and tracked using the platform included activity, nutrition, weight, mood, blood pressure, heart rate, glucose, and oxygen levels. All numerical data could be visualized by the participant in either a trend or table format. iWellness also contained a virtual journal whereby participants could enter and track information about their life moments and experiences and a resource section that provided links to credible information and support related to dementia. Finally, the platform provided users with an opportunity to connect with other iWellness users via the "My Team" and "My Messages" features. 


\subsection{Measures}

\subsubsection{Demographic questionnaire}

A brief demographic questionnaire was administered to all participants to collect information about age, gender, potential health concerns and issues, and the relationship of the caregiver to the person with dementia.

\subsection{2. iWellness semi-structured interview guide}

Semi-structured in-person interviews were conducted, and questions were asked to elicit participants' opinions, experiences, and feedback about using the platform in conjunction with a Fitbit device (e.g., "What features do you use the most?", "What additional information could we collect and/or include that would be useful to you?").

\subsubsection{Procedure}

Approval to conduct the research was obtained from the appropriate Research Ethics Boards. Participants were recruited through social media, community-based service programs for individuals with dementia (e.g., Day Care Programs, Alzheimer's Café), and radio/bulletin board announcements. Interested participants could sign up or request more study information by contacting one of the primary investigators or research assistants by phone or e-mail. One of two research assistants arranged to meet with each participant at a mutually convenient time, date, and location. Research assistants (RAs) met with all caregiver participants at baseline and at 4 and 12 weeks after participant enrolment.

To be eligible, participants were required to be a primary caregivers of and living with an individual with a diagnosis of dementia. Participants also had to be open to adopting new technology, have internet in their homes, and be able to communicate in English as the iWellness platform was only available in English. After the RA had ensured that all eligibility criteria were met, informed consent was obtained. Participants were informed that all answers were confidential and that they could withdraw from the study at any time.

After obtaining informed consent, a RA demonstrated the iWellness platform and answered any questions from participants throughout a one-on-one training session. Following the introduction to the iWellness program, a Fitbit Flex 2 was set up and synced with the iWellness program so that information on steps and sleep was automatically uploaded to the program. The RAs also identified if caregivers had a need or desire for additional monitoring equipment (e.g., glucometer, smart scale, blood pressure monitor) that would enhance participants' health and well-being. Together, the RA and participant determined which devices from a select group of health monitoring devices were relevant to the caregiver's health concerns and communicated this information to the research team. Only one participant expressed a desire for additional monitoring equipment (i.e., blood pressure monitor); the research team determined that there was a sufficient anticipated benefit and supplied the additional equipment to this participant. This participant was provided with individualized instruction on the use of this health monitoring device.

Additionally, participants were invited to join a closed Facebook caregiver support group (i.e., iWellness Caregiver Support group) where they had the opportunity to connect with other caregivers recruited to the current study. The iWellness device provided to participants had the capability of connecting to the Facebook site. At the training session, the RA encouraged participants to reach out if they had any inquiries about the iWellness program, 
Fitbit, or the study in general and provided participants with contact information (phone and e-mail address).

\subsection{Data collection}

One-on-one semi-structured interviews were conducted with participants at 4 and 12 weeks following enrollment into the study. The RA met with each participant at a mutually convenient time at a location chosen by the participant. Except for three, all interviews took place in the participant's home. Twenty participants were interviewed during the four weeks and 15 interviews were conducted during the 12 weeks for a total of 35 interviews. Of the participants who did not complete the second interview, three were unavailable due to personal reasons, while the family member of the others had moved into a nursing home so the participants declined the interview. Interviews ranged from 20 to 50 minutes. All interviews took place between April 2017 and November 2017. During the interviews, participants were asked about their experience with using the iWellness platform in conjunction with the Fitbit Flex 2.

\subsection{Data analysis}

Transcribed interview data were analyzed using Braun and Clarke's [31] approach to thematic analysis. The interview data were reviewed independently and on multiple occasions by members of the research team and discussed during team meetings until all the major themes were identified. During these meetings, members shared their preliminary codes and discussed these among the group until all common codes were agreed upon. Where disagreement occurred, researchers reviewed the transcripts to determine how well the data supported the codes and themes. This continued until a consensus was reached among all research team members. Lincoln and Guba's four criteria were used to assess the trustworthiness of the data: credibility, transferability, dependability, and confirmability[32]. Credibility was enhanced by investigator triangulation; members of the team analyzed the data independently before having team discussions. To enhance transferability, descriptions of the participants, the data collection and analysis methods, and the results with relevant quotations are provided. To ensure dependability and confirmability, an audit trail was kept to increase the transparency of the research process, including transcripts, data analysis decisions, and interview guides.

\section{Results}

\subsection{Demographics}

A total of 4 male (Mage $=73.33$ years, $S D=5.03)$ and 16 female $($ Mage $=62.12$ years, $S D$ $=12.66)$ family/friend caregivers of persons with dementia from one province in Atlantic Canada participated in the study. The majority of caregivers: identified as the spouse/partner $(66.7 \%)$ of the person they cared for at home (Parent 28.6\%, Family Member 4.8\%); reported not having any publicly funded supports at home $(66.7 \%)$; and, had not considered placing their loved one in a long-term facility $(66.7 \%)$. Over half $(62 \%)$ of participants expressed concerns about their health (high blood pressure $47.6 \%$, diabetes $9.5 \%$, weight $42.9 \%$, lack of exercise $47.6 \%$, breathing problems $14.3 \%$, problems sleeping $47.6 \%$ ). On a six-point Likert scale $(0=$ Not stressful; $5=$ Very stressful $)$, on average, participants reported that caring for a loved one with dementia was a somewhat stressful experience $(M=3.36, S D=1.09)$. 


\subsection{Findings}

Caregivers in this study identified five themes related to their experiences with using the iWellness platform in conjunction with a Fitbit wearable device. These themes included: (1) Becoming Self-Aware; (2) Providing the Motivation; (3) A Place for Reflection and Expression; (4) Competing Priorities Take Precedence; and, (5) Equipment and Technical Issues.

\subsubsection{Becoming self-aware}

Caregivers described how tracking certain data (e.g., blood pressure, glucose, thoughts, and feelings) or monitoring their activity (i.e., number of steps) and/or sleep patterns using the Fitbit and/or iWellness applications helped them become more self-aware of their health. For most participants, this self-awareness provided them with personal information that they otherwise did not consider:

The Steps and Sleep features were the most valuable to me because they made me more aware of how much I was getting. [Participant 7, daughter]

Tracking blood pressure and glucose gives me a way to know where I stand and how my days are going. What is causing it to go up and what is causing it to go down? [Participant 1, spouse].

Probably the recording of sleep has been the most valuable because it helps me get

an accurate perspective that I need to permit myself to have a nap in the afternoon. [Participant 6, spouse].

Many participants reported that before using their mobile wellness applications, they were unaware, of or did not hold an accurate estimate of, certain health indicators. For instance, one caregiver [Participant 9, spouse] stated "I like it [monitoring Sleep and Steps] because my perceptions are not always what the reality is." Several participants described how the act of tracking information about their health provided them with the opportunity to focus on themselves and forced them to pay more attention to their well-being while caring for their loved ones.

"The Fitbit and iWellness have given me something that makes me focus on me." [Participant 8, daughter].

"The information available on iWellness makes me pay more attention, I pay attention to activity and my sleep." [Participant 17, daughter].

"Seeing the steps is making me more aware of my health. It's making me think that I have to take care of myself." [Participant 20, spouse].

\subsubsection{Providing the motivation}

For some caregivers, viewing and monitoring their personal health information provided them with the motivation necessary to implement changes in their own lives. Having access to data on activity levels and sleep patterns was affirming and provided an entry point to which they could share some of their day-to-day struggles associated with caregiving. Data on sleep patterns and activity in particular highlighted specific areas where their caregiving role was compromising their well-being. At times, this awareness facilitated changes in their own lives and the use of support services.

It has made me make some changes. It's a right there-in-your-face kind of thing. I have enrolled my husband for an additional day in a daycare program and taken the day off so that 
I can come home and rest. I have started implementing changes in my life. I am going to guard the extra day as a 'me' day. [Participant 6, spouse]

Tracking my sleep is what I found the most helpful because that is so important, getting the proper sleep. I have taken different steps to ensure that I am more rested. I feel that I have more energy now and am more capable of dealing with things. When you're overtired everything is more difficult. I can now handle my emotions a lot better. [Participant 8, daughter].

I had no idea how many steps I took in a day. I found myself more willing to do things that took more steps. It was a motivational thing. [Participant 10, daughter].

\subsubsection{A place for reflection and expression}

Over half $(60 \%)$ of the participants utilized the journal feature on the iWellness platform and found it to be therapeutic. Participants described the journal as offering a safe place to express their feelings privately and to help them keep track of daily events. Taking the time to reflect on their experiences was self-affirming and provided some participants with a greater awareness of the multitude of things they accomplish as a caregiver and increased acceptance of their current life circumstances. Furthermore, several participants acknowledged that the time spent journaling and reflecting was time they would not have otherwise spent focusing on themselves.

The journal was one of the features that provided the most value to me. I was able to get things off my chest. I was able to vent without standing in a room and screaming. [Participant 1 , spouse].

"It [the journal] just gives me the chance to blabber on. It's a stress reliever. Just let your emotions flow." [Participant 16, spouse].

The journal was the most valuable feature because it made me take the time to do it and normally I wouldn't have. It also keeps your memory alert because you can look back and see what took place and what you have or have not accomplished. [Participant 4, spouse].

Aside from the journal feature, participants also described how using the iWellness platform allowed them to take the time to reflect and think about themselves during the day. For instance, one participant [Participant 4, spouse] stated, "It [iWellness] allows me to think about myself for an hour or so. Otherwise, I would not take that time." Another participant [Participant 9, spouse] reported, "I think it [iWellness] has made me look at myself a little more, which is a good thing."

\subsubsection{Competing priorities take precedence}

A few participants reported that they did not use the iWellness application often due to time constraints, or they felt it was not beneficial for them to manually track certain personal health information. However, even though a few participants did not regularly use the iWellness platform, they still described how viewing information on their sleep and activity patterns (i.e., number of steps) on the Fitbit application was beneficial for them. Ultimately, a few participants did not perceive the iWellness was beneficial, either because of competing priorities or the need for them to shift their focus from their caregiving duties to themselves.

It was hard to commit to using the program because of time restraints. My schedule is all over the place for work and caring for my mother. [Participant 2, daughter].

There's a bunch of iWellness I didn't use, didn't want to use, or didn't have the means to input the data. If you tracked everything, it would take an hour. [Participant 16, spouse]. 
It [iWellness] is too introspective and self-focused, I need to be focused on other people. I find you can get a little excessive with this stuff. I feel best when I am looking after and caring for people. [Participant 12, spouse].

\subsubsection{Equipment and technical issues}

Some participants were challenged with using the technology, but most of these issues were addressed with support from the RAs. The technical issues experienced with wearable devices included: 1) data syncing problems; 2) charging the device; and, 3) concerns with comfort and fastening of the armband on the FitBit. The technical issues experienced with using the iWellness platform included: 1) logging in; 2) navigating the platform; 3) inputting data; and, 4) syncing data from wearable devices such as the Fitbit with the platform. I forgot to charge it every three days. I also had problems with the data synching to my device the first week. [Participant 12, spouse].

I got discouraged with using the Fitbit. There were synching problems. This past month I do not think there is any information there. Even if it did sync, the information would disappear a day later. I kept making sure it was charged and to see if it was synching but I do not have time to troubleshoot the problems. I do not have the mental energy for it. It is another source of frustration that I do not have time to be bothered with. [Participant 6, spouse].

\section{Discussion}

This study explored the experiences of family caregivers who used an online wellness platform (iWellness; Inflection Point Healthcare, Canada) that supports multiple metrics for education, support, and engagement, in conjunction with wearable health monitoring devices. Findings are in line with previous research findings in this area which suggest that internet interventions can help caregivers cope with the various aspects of the caregiving role. [24][33] Data gathered from semi-structured interviews indicated that despite having technology issues, the iWellness platform helped caregivers engage with their health and supported them in their caregiving role.

Participants in this study described how having a snapshot of their health data allowed them to become more self-aware and engaged in their health and well-being. Many caregivers reported that they had been previously unaware of certain aspects of their personal health information (e.g., activity, sleep patterns), and that being able to view and monitor this information allowed them to recognize patterns and implement changes to better their life circumstances. For example, several caregivers were not aware of their severe lack of sleep and took concrete steps to ensure they were more rested and better able to cope with the everincreasing demands, responsibilities, and stress placed upon them in their caregiving roles. Sleep disturbances are common amongst caregivers of people with dementia and are often associated with concerns about the loved one wakening at night, wandering, and falling. [34] Sleep disruption can have considerable short and long-term health consequences including reduced quality of life, fatigue, emotional distress, hypertension, cardiovascular disease, type 2 diabetes, and colorectal cancer [35]. Caregiver sleep problems have also been noted to play a role in decisions about institutionalization [36]. Identifying problems with sleep is an important finding in this research as it prompted caregivers to identify strategies to improve sleep and avoid unnecessary health problems. The participants' attention given to their identified sleep problems may assist them in keeping their loved ones home for a longer period. 
Several caregivers viewed the iWellness platform as an opportunity to focus on themselves, in addition to the usual act of focusing on the health and well-being of the person they were caring for at home. Caregivers described the journaling capability of the platform as a place for reflection and personal expression, and found it an effective avenue to "vent", "get things off their chest", and/or "keep track of daily events and memories". Consistent with the findings of Kidd, Zauszniewski, and Morris [37], it is possible that the device offered a "welcomed distraction" from the constant concern about their loved one's health and safety. The therapeutic value of journaling within the platform should not be underestimated. Caregivers of individuals with dementia are often socially isolated and have limited opportunities to share their struggles with others [38]. As noted in this study, there may be value in caregivers reflecting on their caregiving experiences, particularly in situations when their circumstances preclude them from reaching out to others. The use of reflective journaling for dementia caregivers is an area where further research is required.

Some caregivers expressed concern that the time required to use the iWellness platform and Fitbit would take them away from their caregiving duties. This finding is consistent with previous research that reports caregivers often describe themselves as being too busy or overwhelmed to seek support to assist them in their roles [39]. The time required to access resources or learn new skills often overshadows any potential benefits, such as reduced frustration and increased caregiving efficiencies. Moreover, some caregivers may be embarrassed to ask for assistance or fear being perceived as inadequate if they ask for assistance in using a resource. [39] When introducing resources to caregivers, it is important for healthcare providers and community agencies to be mindful of perceived time barriers. This is especially true now when the landscape of community resources is changing and becoming more technologically based [24][26][40]. While these technology-based resources offer great promise, particularly for those caregivers finding it difficult to leave their homes or require flexibility in using resources, not all caregivers may be willing to engage in the technology. Caregivers must have access to a range of supports that meet their unique needs and preferences.

\section{Limitations}

Although this study provides valuable information about caregivers' experiences with a technological platform and health monitor devices, it has several limitations that should be noted. Participant experiences were only sought after engaging with the platform for a short period of 12 weeks. It is possible that participants' long-term experiences differed substantially, particularly after the novelty of having a new device wore off. Additionally, it is unknown if the platform's ability to make participants more aware of their health risks translated into positive lifestyle changes. Participants in this study were largely female, were not receiving any publically funded community services, and had not yet considered placing their loved one in a long-term care facility. It is possible that the experiences of caregivers living with different circumstances would differ.

\section{Conclusion}

Participants in this study provided valuable insights into how health monitoring devices can be used to assist caregivers when caring for a loved one with dementia. Findings demonstrate that technology and health monitoring devices can help caregivers become mindful of their health and can even motivate them to make positive changes in their lives. Eventually, these changes may help to maintain caregivers' health and possibly reduce 
healthcare expenditures by reducing their health risks and premature need for institutional care for their loved ones. However, the overwhelming demands placed on caregivers may prevent them from learning how to use technology or from appreciating how technology can assist them in their roles. Healthcare providers and community agencies need to raise awareness about how technology can assist caregivers in maintaining their health, and help caregivers overcome the challenges to its acceptance and use.

\section{References}

[1] World Health Organization, "Global action plan on the public health response to dementia 2017-2025," World Health Organization, (2017)

[2] World Health Organization, Dementia: Key facts, (2019)

[3] H. Chertkow, "An action plan to face the challenge of dementia: international statement on dementia from IAP for health,” JPAD, vol.3, pp.207-212, (2018)

[4] Canadian Institute of Health Information, Dementia in Canada, (2018)

[5] B. Black, et al., "Unmet needs in community-living persons with dementia are common, often non-medical and related to patient and caregiver characteristics," Int Psychogeriatr, vol.31, pp.1-12, (2019)

[6] C. Whitlatch and S. Orsulic-Jeras, "Meeting the informational, educational, and psychosocial support needs of persons living with dementia and their family caregivers," Gerontologist, vol.58, pp.S58-S73, (2018)

[7] Xiong, G., et al., "The communication gap between the medical system and community resources for dementia-related behavioral symptom management: Family caregiver perspectives," Prim Care Companion CNS Disorder, vol.20, no.2, (2018) DOI: 10.4088/pcc.17m02238

[8] C. Morrisby, A. Joosten, and M. Ciccarelli, "Do services meet the needs of people with dementia and carers living in the community? A scoping review of the international literature," Int Psychogeriatric, vol.30, pp.514, (2018)

[9] S. Weber, P. Pirraglia, and M. Kunik, "Use of services by community-dwelling patients with dementia: a systematic review,” Am J Alzheimer's Dis Other Demen, vol.26, pp.195-204, (2011)

[10] L. Phillipson, S. Jones, and C. Magee, "A review of the factors associated with the non-use of respite services by carers of people with dementia: implications for policy and practice," Health Soc Care Community, vol.22, pp.1-12, (2014)

[11] L. Boots et al, "Qualitative study on needs and wishes of early-stage dementia caregivers: The paradox between needing and accepting help," Int Psychogeriatr, vol.27, pp.927-936, (2015)

[12] R. Herron and M. Rosenberg, "Not there yet": Examining community support from the perspective of people with dementia and their partners in care," Soc Sci Med, vol.173, pp.81-87, (2017)

[13] A. Macleod, et al., "There isn't an easy way of finding the help that's available" Barriers and facilitators of service use among dementia family caregivers: A qualitative study," Int psychogeriatrics, vol.29, no.5, pp.765-776, (2017) DOI: 10.1017/S1041610216002532

[14] K. Robinson, K. Buckwalter, and D. Reed., "Differences between dementia caregivers who are users and nonusers of community services," Public Health Nurs, vol.30, pp.501-510, (2013)

[15] H. Brodaty, "Why caregivers of people with dementia and memory loss don't use services," Int J Geriatr Psychiatry, vol.20, pp.537-546, (2005)

[16] J. Bryant, "Characteristics of the informal caregiver: An integrative literature review," Walden Dissertations and Doctoral Studies, (2016)

[17] J. Millenaar, et al., "The care needs and experiences with the use of services of people with young - onset dementia and their caregivers: A systematic review,” Int J Geriatric Psychiatry, vol.31, no.12, pp.1261-1276, (2016) DOI: $10.1002 /$ gps.4502 
[18] J. Røsvik, et al., "Interventions to enhance access to and utilization of formal community care services for home-dwelling persons with dementia and their informal careers, A scoping review," Aging Ment Health, vol.23, pp.1-12 (2019)

[19] B. Suehs, B., et al., "Household members of persons with Alzheimer's disease: Health conditions, healthcare resource use, and healthcare costs," J Am Geriatr Soc, vol.62, pp.435-441, (2014)

[20] A. Koyama, A., "Mental health among younger and older caregivers of dementia patients," psychogeriatrics, vol.17, pp.108-114, (2107)

[21] P. Cuijpers, "Depressive disorders in caregivers of dementia patients: A systematic review," Aging Ment Health, vol.9, pp.325-330, (2005)

[22] L. Contador, et al., "Prediction of burden in family caregivers of patients with dementia: A perspective of optimism based on generalized expectancies of control," Aging Ment Health, vol.16, pp.675-682, (2012)

[23] Y. Liu, Y., K. Kim, and S. Zarit, "Health trajectories of family caregivers: Associations with care transitions and adult day service use," J Aging Health, vol.27, pp.686-710, (2015)

[24] L. Boots, et al., "A systematic review of Internet - based supportive interventions for caregivers of patients with dementia," Int J Geriatr Psychiatry, vol.29, pp.331-344, (2014)

[25] F. Meichsner, C. Theurer and G. Wilz, "Acceptance and treatment effects of an internet-delivered cognitive - behavioral intervention for family caregivers of people with dementia: A randomized controlled trial," J Clin Psycho, vol.75, pp.594-613, (2019)

[26] J. Hopwood, et al., "Internet-based interventions aimed at supporting family caregivers of people with dementia: Systematic review,” J Med Internet Res, vol.20, no.6, pp.e216, (2018) DOI: 10.2196/jmir.9548

[27] G. D'Onofrio, et al., "Information and communication technologies for the activities of daily living in older patients with dementia: A systematic review," J Alzheimers Dis, vol.57, pp.927-935, (2017)

[28] D. Parker Oliver, et al., "The effect of internet group support for caregivers on social support, self-efficacy, and caregiver burden: a meta-analysis," Telemed E-Health, vol.23, pp.621-629, (2017)

[29] E. Chiauzzi, C. Rodarte, and P. DasMahapatra, "Patient-centered activity monitoring in the self-management of chronic health conditions," BMC Med, 13, article no.77, (2015)

[30] Wang, K., G. Chen, and H. Chen, "Understanding technology adoption behavior by older adults," Soc Behav Pers, vol.46, pp.801-814, (2018)

[31] V. Braun, V. and V. Clarke, "Using thematic analysis in psychology," Qual Res Psychol 2006, vol.3, pp.77101, (2006)

[32] Y. Lincoln and E. Guba, "But is it rigorous? Trustworthiness and authenticity in naturalistic evaluation," In DD Williams, ed. Naturalistic Evaluation, Jossey-Bass, San Francisco, CA., pp.73-84, (1985)

[33] J. García-Casal, et al., "Computer-based cognitive interventions for people living with dementia: a systematic literature review and meta-analysis," Aging Ment Health, vol.21, pp.454-467, (2017)

[34] A. Leggett, A., et al., "What hath night to do with sleep?: The caregiving context and dementia caregivers' nighttime awakenings," Clin Gerontol, vol.41, pp.158-166, (2018)

[35] G. Medic, M. Wille, and M. Hemels, "Short and long-term health consequences of sleep disruption," Nat Sci Sleep, vol.9, pp.151, (2017)

[36] Ancoli-Israel, S. and M. Vitiello, "Sleep in dementia," Am J Geriatr Psychiatry, vol.14, pp.91, (2006)

[37] L. Kidd, J. Zauszniewski and D. Morris, "Benefits of a poetry writing intervention for family caregivers of elders with dementia,” Issues Ment Health Nurs, vol.32, pp.598-604, (2011)

[38] [M. McCabe, E. You, and G. Tatangelo, "Hearing their voice: A systematic review of dementia family caregivers' needs," Gerontologist, vol.56, pp.e70-e88, (2016)

[39] K. Peterson, et al., "In the Information Age, do dementia caregivers get the information they need? Semistructured interviews to determine informal caregivers' education needs, barriers, and preferences," BMC Geriatr 1-13, pp.164, (2016)

[40] C. Hu, et al., "Reducing caregiver stress with internet-based interventions: A systematic review of open-label and randomized controlled trials," J Am Med Inform Assn, vol.22, pp.e194-e209, (2014) 


\title{
Authors
}

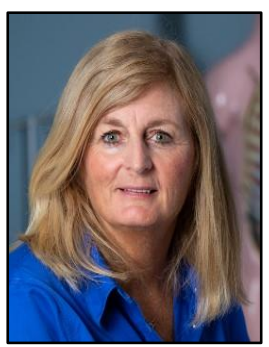

\author{
Dr. Rose McCloskey \\ Professor, Nursing \& Health Sciences \\ University of New Brunswick \\ New Brunswick, Canada
}

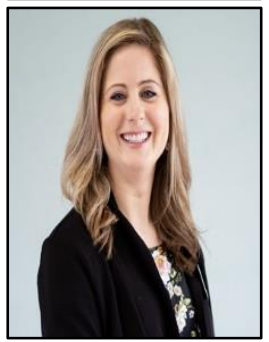

\section{Ms. Leanne Skerry}

Research Coordinator

Horizon Health Network

New Brunswick, Canada

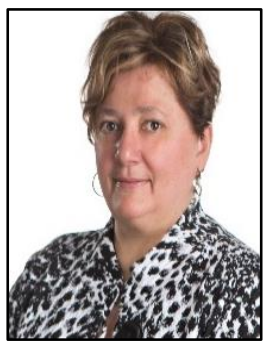

\section{Dr. Lisa Keeping-Burke}

Associate Dean of Health Research

The University of New Brunswick,

Saint John, Canada

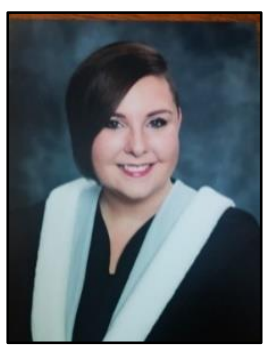

\section{Ms. Alicia Donovan}

Teacher at the Anglophone South School District

New Brunswick, Canada

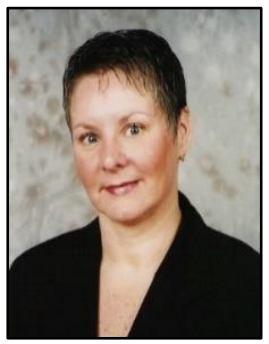

\section{Ms. Cindy Donovan}

Chief Executive Officer Loch Lomond Villa Inc.

Saint John New Brunswick, Canada

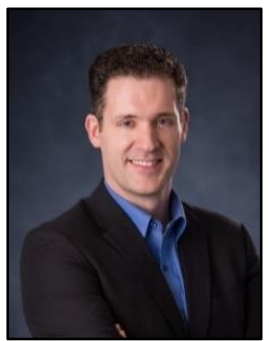

\section{Dr. Eric Scheme}

NB Innovation Research Chair in Medical Devices

and Technologies and Associate Professor

Electrical and Computer Engineering

University of New Brunswick

Fredericton, Canada 